\title{
Fish consumption and the risk of myocardial infarction and stroke in the German arm of the European Prospective Investigation into Cancer and Nutrition (EPIC-Germany)
}

\author{
Tilman Kühn $^{1 *}$, Birgit Teucher ${ }^{1}$, Rudolf Kaaks ${ }^{1}$, Heiner Boeing ${ }^{2}$, Cornelia Weikert ${ }^{2}$ and Brian Buijsse ${ }^{2}$ \\ ${ }^{1}$ Division of Cancer Epidemiology, German Cancer Research Center (DKFZ) Heidelberg, Im Neuenheimer Feld 581, 69120 \\ Heidelberg, Germany \\ ${ }^{2}$ Department of Epidemiology, German Institute of Human Nutrition (DIfE) Potsdam-Rehbrücke, Arthur-Scheunert-Allee \\ 114-116, 14558 Nuthetal, Germany
}

(Submitted 30 August 2012 - Final revision received 4 January 2013 - Accepted 5 January 2013 - First published online 15 February 2013 )

\begin{abstract}
Recent meta-analyses have confirmed that fish consumption is related to decreased risks of ischaemic stroke and fatal CHD, while there seem to be no clear associations between fish consumption and the risks of haemorrhagic stroke and non-fatal CHD. As no studies in German populations have been reported to date, we assessed whether fish consumption as recorded by FFQ between 1994 and 1998 was related to incident myocardial infarction (MI) and stroke within the German arm of the European Prospective Investigation into Cancer and Nutrition (EPIC) study. Cox proportional hazards regression analyses were conducted based on the data of 48315 participants aged 35-65 years at baseline. The median fish intake was $16 \cdot 4 \mathrm{~g} / \mathrm{d}$ (25th-75th percentile $8 \cdot 2-28 \cdot 8 \mathrm{~g} / \mathrm{d})$. During a mean follow-up of 8.1 years, 605 incident MI and 525 incident strokes have been documented. After multiple adjustment, fish consumption was not related to incident MI (hazard ratio (HR) $0.84,95 \%$ CI $0 \cdot 66,1 \cdot 08, P_{\text {trend }}=0 \cdot 21$ ) or stroke (HR 0.96, 95\% CI $0.73,1 \cdot 26, P_{\text {trend }}=0 \cdot 67$ ). Separate analyses for fatal MI, ischaemic stroke and haemorrhagic stroke did not show significant associations, either. With regard to non-fatal MI, there was a non-significant trend for an inverse association (HR 0.78, 95\% CI 0.59, 1.03, $P_{\text {trend }}=0.07$ ). Overall, fish consumption was not related to the risks of MI and stroke in the EPIC-Germany study.
\end{abstract}

Key words: Fish consumption: CVD: Myocardial infarction: Stroke

CVD are the leading cause of death worldwide(1). They are considered 'highly preventable,(2), particularly by the modification of dietary and lifestyle factors ${ }^{(2-4)}$. A diet characterised by a regular consumption of fish is recommended for the prevention of both $\mathrm{CHD}$ and stroke $\mathrm{e}^{(3-6)}$. Meta-analyses of observational studies have shown that a high habitual fish consumption is associated with lower risks of $\mathrm{CHD}^{(7,8)}$ and stroke $^{(9,10)}$. Interestingly, there seems to be no clear association between fish consumption and the risk of non-fatal $\mathrm{CHD}^{(6,11)}$, whereas fish consumption seems to be strongly related to a decreased risk of fatal $\mathrm{CHD}^{(8)}$. This difference has been attributed to the anti-arrhythmic properties of $n$ - 3 fatty acids ${ }^{(11-14)}$. The association between fish consumption and stroke risk seems to depend on stroke subtype. While a recent meta-analysis revealed a clear inverse association between fish consumption and the risk of ischaemic stroke, there was no significant association with the risk of haemorrhagic stroke ${ }^{(9)}$. An anti-thrombotic effect of $n-3$ fatty acids as the underlying mechanism particularly protecting from ischaemic stroke has been proposed ${ }^{(6)}$.

Besides anti-arrhythmic and anti-thrombotic mechanisms, anti-inflammatory, antioxidative and hypolipidaemic as well as heart rate and blood pressure-lowering effects of marine $n-3$ fatty acids, i.e. EPA and DHA, have been described $^{(11,13,15,16)}$. Some of the suggested effects might be based upon a combination of nutrients in fish acting in synergy rather than $n-3$ fatty acids alone ${ }^{(6)}$. Notably, fish is an important dietary source of vitamin $\mathrm{D}^{(17)}$ whose cardioprotective properties are being extensively researched ${ }^{(18)}$. Also, inverse associations between fish consumption and the risks of CHD and stroke may be partly explained by the fact that fish consumption is related to an overall healthconscious lifestyle and a higher socio-economic status $(6,19,20)$.

International fish intake recommendations for general populations suggest at least one or two servings of preferably oily fish per week ${ }^{(3-5,11)}$. These recommendations correspond

Abbreviations: EPIC, European Prospective Investigation into Cancer and Nutrition; HR, hazard ratio; MI, myocardial infarction. 
with the $\mathrm{FAO} / \mathrm{WHO}$ recommendation for healthy people to consume at least $250 \mathrm{mg}$ of marine-derived $n$-3 fatty acids, i.e. DHA and EPA combined per $\mathrm{d}^{(11,21)}$. Fish consumption in Germany is low compared with other European countries according to market data ${ }^{(22)}$. Also, previous results from the European Prospective Investigation into Cancer and Nutrition (EPIC) showed that median fish intakes as assessed by FFQ at the German study centres Heidelberg (women $15 \mathrm{~g} / \mathrm{d}$, men $16 \mathrm{~g} / \mathrm{d}$ ) and Potsdam (women $16 \mathrm{~g} / \mathrm{d}$, men $23 \mathrm{~g} / \mathrm{d}$ ) were low compared with the overall median intakes at twenty-four European study centres (women $29 \mathrm{~g} / \mathrm{d}$, men $29 \mathrm{~g} / \mathrm{d}$ ) ${ }^{\text {(23) }}$.

The prospective association between fish consumption and the risks of CHD and stroke has not been explored in a general German population to date, and previous findings suggested that even a moderate fish consumption may lead to a reduction of both CHD mortality and stroke risk ${ }^{(8-10)}$. We therefore investigated the relationship of fish consumption and the risk of myocardial infarction (MI) and stroke within the German arm of the EPIC study. We hypothesised that fish consumption would be inversely associated with the risks of MI and stroke, with stronger associations for fatal MI and ischaemic stroke compared with non-fatal MI and haemorrhagic stroke, respectively.

\section{Methods}

\section{Study population}

EPIC-Germany is part of the multi-centre European EPIC study and comprises two independent cohorts at centres in Heidelberg and Potsdam ${ }^{(24)}$. Between 1994 and 1998, 25540 participants (13612 women and 11928 men) in Heidelberg and 27548 participants (16644 women and 10904 men) in Potsdam aged mostly between 35 and 65 years were recruited from the local general population. Participation rates were $22.7 \%$ in Potsdam and $38.3 \%$ in Heidelberg. Comparisons with reference populations revealed that subjects who entered the study were characterised by a higher socio-economic status and lower prevalences of obesity and hypertension ${ }^{(24)}$. Baseline examinations included detailed self-administered questionnaires and interviews on health status, socio-economic status and lifestyle, anthropometric measurements and a comprehensive dietary assessment by a validated self-administered $\mathrm{FFQ}^{(24)}$. Participants were followed up by active and passive procedures ${ }^{(25)}$. For the present study, we included data from complete follow-up rounds up to the end of December 2006. The study was approved by the ethics committees of the Medical Society of the federal state of Brandenburg (Potsdam) and the Faculty of Medicine at the University of Heidelberg. All participants gave written informed consent.

\section{Assessment of fish consumption}

Habitual dietary intake in the year preceding the baseline examination was assessed by a 148-item FFQ. There were ten given frequencies ranging from 'never' to 'five times a day/more' that could be combined with pre-specified serving sizes, allowing for a calculation of intakes in $\mathrm{g} / \mathrm{d}$. The following two items on fish were part of the FFQ: fish as the main component of a warm meal (fried fish, boiled fish and fish fingers) and fish as the component of a cold meal (canned fish and smoked fish). Total fish consumption, the main exposure variable in the present study, was calculated by summing these two fish items. Details on the design and reproducibility of the FFQ have been described elsewhere ${ }^{(26-28)}$. In brief, the FFQ showed good reproducibility of total fish consumption after 6 months (Spearman's correlation coefficient $\rho=0.77)^{(26)}$ and a moderate long-term reproducibility after 69 months $(\rho=0.52)^{(26,28)}$.

\section{Case ascertainment}

We ascertained incident cases of MI and stroke by medical verification of self-reports of incident disease from questionnaires sent to the participants about every 2 years during follow-up and death certificate data. Only cases that were confirmed by our study physicians reviewing medical records provided by treating physicians and hospitals were included into the present study. According to the criteria of the Monitoring Trends and Determinants of Cardiovascular Disease (MONICA) project ${ }^{(29)}$, end points were classified as fatal in the case of death within the first $28 \mathrm{~d}$ after diagnosis or when verified by death certificate. The following end points were coded according to the International Statistical Classification of Diseases, 10th revision, 2011 (ICD-10): MI (ICD-10 I21); ischaemic stroke (ICD-10 I63); haemorrhagic stroke (ICD-10 I60 and I61); unspecified stroke (ICD-10 I64).

\section{Exclusion criteria}

For the present analyses, we applied the following exclusion criteria: (1) baseline age outside the age range pre-specified in the core study protocol, i.e. ages $<35$ and $>65$ years; (2) prevalent MI or stroke; (3) no follow-up data; (4) missing information on stroke and/or MI at follow-up; (5) reporting of implausible energy intakes, i.e. values in the top or bottom $1 \%$ of the ratio of energy intake over energy requirement; (6) missing baseline covariate values.

\section{Statistical evaluation}

Fish consumption values $(\mathrm{g} / \mathrm{d}$ ) were adjusted for energy intake by the residual method ${ }^{(30)}$. The residual values were added to the overall population mean of fish consumption. Descriptive analyses adjusted for age and sex were performed according to quintiles of energy-adjusted fish consumption levels. Partial Spearman's correlation coefficients adjusted for age and sex were calculated to assess the relationship between fish consumption and energy-adjusted dietary covariates.

The associations of fish consumption with MI and stroke were assessed by computing hazard ratios (HR) using Cox proportional hazards regression. Fish consumption was divided into quintiles. The following end points were used as outcome parameters: total MI; fatal MI; non-fatal MI; 
total stroke; ischaemic stroke; haemorrhagic stroke. Participants with both incident stroke and incident MI ( $n$ 23) were excluded from the analyses of the chronologically second event occurring.

Age was the underlying time scale in Cox regression models defining entry and exit time by the participants' age at baseline and the latest follow-up date or the date of disease incidence, respectively. We allowed the baseline function to vary by age (1-year integers) and study centre. The following three Cox regression models were built: model 1 was adjusted for sex. Model 2 was additionally adjusted for classical CVD risk factors, i.e. BMI ('normal weight', $<25 \mathrm{~kg} / \mathrm{m}^{2}$; 'overweight', $25-29.99 \mathrm{~kg} / \mathrm{m}^{2}$; 'obese', $>30 \mathrm{~kg} / \mathrm{m}^{2}$ ), waist circumference (cm, continuous), educational attainment ('low', primary school or no school graduation; 'medium', secondary, technical or professional school graduation; 'high', longer education/university graduation), smoking ('never', 'former', '1-20 cigarettes/d', '> 20 cigarettes/d'), physical activity according to the Cambridge Physical Activity Index ${ }^{(31)}$ ('active', 'moderately active', 'moderately inactive', 'inactive'), alcohol consumption ( $\mathrm{g} / \mathrm{d}$, continuous), prevalent diabetes and energy intake $(\mathrm{kJ} / \mathrm{d}$, continuous). Model 3, finally, was further adjusted for the following dietary factors related to the risk of CVD: fruit, vegetable and meat consumption ( $\mathrm{g} / \mathrm{d}$, continuous). However, because the results from model 3 did not differ from those of model 2 and because fish consumption was only weakly related to other food groups, we used model 2 as our main multivariable-adjusted model.

We did not adjust for prevalent self-reported hypertension, as hypertension may be an intermediate factor on the aetiological pathway between fish consumption and MI and stroke, respectively ${ }^{(6)}$. Instead, we conducted sensitivity analyses stratifying participants with and without hypertension at baseline. Further sensitivity analyses were carried out, excluding participants with prevalent cancer, or participants with prevalent diabetes, or participants with an incident MI or stroke in the first 2 years of follow-up, or fish non-consumers.

Tests for linear trends were conducted by assigning the energy-adjusted median intake value to each quintile of fish consumption and modelling these values as a continuous variable. Heterogeneity in the HR of MI and stroke between covariate strata was assessed by the log-likelihood ratio test comparing Cox regression models with and without interaction terms of fish consumption $(\mathrm{g} / \mathrm{d}$, continuous) and age, sex, centre, BMI, smoking, physical activity, prevalent hypertension and alcohol intake. Alcohol consumption was categorised for interaction testing (non-consumers; women: $>0-6,>6-12$ and $>12 \mathrm{~g} / \mathrm{d}$; men: $>0-12,>12-24$ and $>24 \mathrm{~g} / \mathrm{d})$. Ages at baseline $(<45,46-55$ and $>56$ years $)$ were also grouped. The rationale for these interaction tests was the notion that fish consumption may particularly protect from MI and stroke in subjects at a higher risk for these conditions, e.g. those with hypertension, diabetes or low socio-economic status. Cox proportional hazards regressions with restricted cubic splines were applied to check for non-linear associations between fish consumption and CVD end points, placing knots at the cut-points of fish consumption quintiles ${ }^{(32,33)}$. SAS 9.2 (SAS Institute) was used for all analyses. Statistical tests were two-sided and $P$ values of $<0.05$ were considered to be statistically significant.

\section{Results}

A total of 53088 participants attended the baseline examinations. After exclusion of participants outside the pre-specified baseline age range ( $n$ 265), with prevalent MI or stroke ( $n$ 1516) without any follow-up information ( $n$ 1398), with missing follow-up information on MI or stroke ( $n$ 510), with implausible energy values ( $n$ 958), or with missing baseline covariates ( $n$ 126), the final study sample included 48315 participants (58.0\% women) with a mean age of 50.5 (SD 8.5) years. During a mean follow-up duration of $8 \cdot 1$ years (SD 1.6; 393556 person-years), 605 incident MI cases and 525 incident stroke cases were verified. It was found that 117 of the MI cases and fifty-nine of the stroke cases were fatal. Of the stroke cases, 407 were classified as ischaemic and ninety-five as haemorrhagic (twenty-three cases could not be specified).

Baseline characteristics according to fish consumption are shown in Table 1 . The median consumption of fish was $16.4 \mathrm{~g} / \mathrm{d}$ (25th-75th percentile $8.2-28.8 \mathrm{~g} / \mathrm{d})$ before and $18.0 \mathrm{~g} / \mathrm{d}$ (25th-75th percentile $9.3-28.2 \mathrm{~g} / \mathrm{d}$ ) after energy adjustment. Only 1497 participants (3.1\%) did not eat fish. The proportions of women in the highest and the lowest fish consumption quintiles were lower than the overall proportion. Fish consumption was positively related to age, overweight and obesity, and to prevalent hypertension and diabetes, while the proportion of current heavy smokers ( $\geq 20$ cigarettes/d) was lower at higher fish consumption levels. Partial Spearman's correlations of fish consumption with vegetable consumption $(\rho=0.09, P<0 \cdot 01)$, fruit consumption $(\rho=0.07, \quad P<0.01)$ and meat consumption ( $\rho=0 \cdot 05, P<0 \cdot 01)$ were weak in magnitude.

The HR of incident MI and stroke by quintiles of fish consumption are shown in Table 2. Fish consumption was not associated with incident MI in the basic (HR between the fifth and the first quintiles: 0.86, 95\% CI 0.68, 1.10, $P_{\text {trend }}=0.21$ ) and the multiple-adjusted model (HR 0.84, $95 \%$ CI $\left.0.66,1 \cdot 08, P_{\text {trend }}=0 \cdot 21\right)$. There was also no association between fish consumption and the risk of fatal MI, neither in the basic nor in the adjusted model (HR $1 \cdot 18$, $95 \%$ CI $\left.0.68,2.06, P_{\text {trend }}=0.37\right)$. For non-fatal MI, both the basic model and the multiple-adjusted model (HR 0.78, 95\% CI $\left.0.59,1.03, P_{\text {trend }}=0.07\right)$ revealed a non-significant trend for an inverse association. Fish consumption was not related to incident overall stroke (HR 0.96, 95\% CI 0.73, 1.26, $\left.P_{\text {trend }}=0.67\right)$, ischaemic stroke (HR 0.87, 95\% CI 0.64, 1.19, $P_{\text {trend }}=0.66$ ) or haemorrhagic stroke (HR 1.46, 95\% CI 0.77 , $\left.2 \cdot 78, \quad P_{\text {trend }}=0 \cdot 16\right)$. Restricted cubic-spline Cox regression analyses did not reveal significant non-linear relationships between fish consumption and any of the CVD end points (data not shown).

Log-likelihood ratio tests showed significant interactions between fish consumption and education level concerning the risk of total MI $(P=0 \cdot 03)$ and total stroke $(P=0 \cdot 01)$. Analyses stratified by education level revealed a significant trend for a positive association between fish consumption and 
Table 1. Baseline characteristics of the study population according to fish consumption quintiles (Q)

\begin{tabular}{|c|c|c|c|c|c|}
\hline \multirow[b]{2}{*}{ Characteristics } & \multicolumn{5}{|c|}{ Fish consumption ( $\mathrm{g} / \mathrm{d}$, limits) ${ }^{*}$} \\
\hline & Q1 $(<7.5)$ & Q2 (7.5-14.5) & Q3 (14.5-21.5) & Q4 (21.5-31.1) & Q5 $(>31 \cdot 1)$ \\
\hline Median fish consumption $(\mathrm{g} / \mathrm{d})^{\star} \dagger$ & $2 \cdot 7$ & $11 \cdot 0$ & $18 \cdot 0$ & $25 \cdot 6$ & $40 \cdot 4$ \\
\hline$n$ & 9663 & 9663 & 9663 & 9663 & 9663 \\
\hline Females (\%) & $50 \cdot 4$ & $64 \cdot 2$ & 62.5 & $59 \cdot 4$ & 53.5 \\
\hline Age (years) & 49.5 & $49 \cdot \overline{8}$ & $50 \cdot 6$ & $51 \cdot 3$ & 51.9 \\
\hline Energy intake $(\mathrm{kJ} / \mathrm{d}) \dagger$ & 9183 & 7573 & 7745 & 7822 & 8177 \\
\hline Alcohol intake $(\mathrm{g} / \mathrm{d}) \dagger$ & 8.7 & 7.6 & 8.5 & 9.0 & $9 \cdot 8$ \\
\hline \multicolumn{6}{|l|}{ Waist circumference $(\mathrm{cm})$} \\
\hline Women & 79.6 & 79.4 & 79.5 & 80.5 & 81.4 \\
\hline Men & 94.7 & 94.2 & 94.2 & $95 \cdot 0$ & $95 \cdot 2$ \\
\hline \multicolumn{6}{|l|}{$\mathrm{BMI}(\%)$} \\
\hline$<25 \mathrm{~kg} / \mathrm{m}^{2}$ (normal weight) & $45 \cdot 9$ & 47.7 & 46.5 & 41.4 & $37 \cdot 0$ \\
\hline $25-29.9 \mathrm{~kg} / \mathrm{m}^{2}$ (overweight) & 38.2 & 38.3 & 38.2 & 41.9 & $43 \cdot 8$ \\
\hline$>30 \mathrm{~kg} / \mathrm{m}^{2}$ (obese) & $15 \cdot 9$ & $14 \cdot 0$ & $15 \cdot 3$ & $16 \cdot 7$ & $19 \cdot 2$ \\
\hline \multicolumn{6}{|l|}{ Smoking (\%) } \\
\hline Never & 43.4 & 47.4 & $48 \cdot 3$ & $47 \cdot 4$ & $45 \cdot 5$ \\
\hline Former & 32.7 & 31.8 & $32 \cdot 1$ & 33.6 & 34.4 \\
\hline Current $(<20$ cigarettes/d) & $14 \cdot 1$ & $13 \cdot 3$ & 13.4 & $12 \cdot 7$ & 13.4 \\
\hline Current $(\geq 20$ cigarettes $/ \mathrm{d}$ ) & 9.8 & 7.5 & 6.2 & $6 \cdot 3$ & $6 \cdot 7$ \\
\hline \multicolumn{6}{|l|}{ Physical activity (\%) } \\
\hline Inactive & $17 \cdot 1$ & 17.4 & $16 \cdot 1$ & $15 \cdot 9$ & $17 \cdot 1$ \\
\hline Moderately inactive & 34.8 & 37.5 & 38.4 & 37.6 & $35 \cdot 6$ \\
\hline Moderately active & $26 \cdot 3$ & 25.9 & $26 \cdot 2$ & $26 \cdot 8$ & $26 \cdot 1$ \\
\hline Active & 21.8 & $19 \cdot 2$ & $19 \cdot 3$ & 19.7 & $21 \cdot 2$ \\
\hline \multicolumn{6}{|l|}{ Educational attainment (\%) } \\
\hline Low level & 24.9 & $22 \cdot 7$ & $22 \cdot 7$ & 23.2 & 23.2 \\
\hline Medium level & 41.9 & $44 \cdot 1$ & 42.5 & $42 \cdot 9$ & $41 \cdot 3$ \\
\hline High level & 33.2 & 33.2 & 34.8 & 33.9 & 35.5 \\
\hline Prevalent hypertension (\%)‡ & $27 \cdot 8$ & $26 \cdot 7$ & 29.9 & $31 \cdot 1$ & 33.3 \\
\hline Prevalent diabetes (\%)‡ & 3.3 & 3.7 & 3.8 & 4.5 & $5 \cdot 3$ \\
\hline
\end{tabular}

stroke in subjects with a high education level $\left(P_{\text {trend }}=0 \cdot 04\right.$; Table 3), while there was no significant difference with respect to extreme quintiles (HR 1.42, 95\% CI 0.84, 2.39). No significant associations depending on education level were detected concerning MI risk, even though there was a nonsignificant trend for an inverse association in participants with a low education level (HR $0 \cdot 67,95 \%$ CI $0 \cdot 44,1 \cdot 03$, $\left.P_{\text {trend }}=0.09\right)$. Further, there were no significant interactions between fish consumption and physical activity $\left(P_{\mathrm{MI}}=0 \cdot 90\right.$, $\left.P_{\text {stroke }}=0.47\right)$, age at baseline $\left(P_{\mathrm{MI}}=0.24, P_{\text {stroke }}=0.90\right)$, smoking $\left(P_{\mathrm{MI}}=0.43, \quad P_{\text {stroke }}=0.36\right)$, alcohol consumption $\left(P_{\mathrm{MI}}=0.58, P_{\text {stroke }}=0.61\right)$, BMI $\left(P_{\mathrm{MI}}=0.29, P_{\text {stroke }}=0.74\right)$, sex $\left(P_{\mathrm{MI}}=0.37, P_{\text {stroke }}=0.56\right)$ or study centre $\left(P_{\mathrm{MI}}=015\right.$, $\left.P_{\text {stroke }}=0.62\right)$ concerning the risk of MI and stroke, or any of the other end points (data not shown). Also, there were no interactions with prevalent hypertension $\left(P_{\mathrm{MI}}=0.34\right.$, $P_{\text {stroke }}=0.34$ ), and separate Cox regression analyses in participants with and without prevalent hypertension did not yield differential associations between fish consumption and the risks of MI or stroke, accordingly (data not shown). Sensitivity analyses excluding fish non-consumers, or prevalent cancer cases, or prevalent diabetes cases, or MI and stroke cases that occurred during the first 2 years of follow-up did not show results diverging from those of the main analyses (data not shown).

\section{Discussion}

In a large middle-aged German population, fish consumption was not related to the overall risks of MI or stroke. While there were no associations between fish consumption and the risks of fatal MI, ischaemic stroke and haemorrhagic stroke, there was a non-significant trend for a linear inverse association concerning the risk of non-fatal MI. Even though significant interactions between fish consumption and education level were detected with regard to both MI and stroke risk, stratified analyses did not show strong relationships between fish consumption and disease risks according to high, medium or low education level.

The present study has limitations. Our results are based on a single assessment of habitual fish consumption by a FFQ that did not include details on preferred fish types and fish preparation methods. However, this is in line with multiple other studies that showed inverse associations between the risks of stroke or MI and total fish consumption rather than fatty fish consumption only ${ }^{(7-9)}$. We did not have sufficient information on the relative validity of our FFQ with regard to fish intake, because a previous validation study using $24 \mathrm{~h}$ recalls did not cover food intake on Fridays, the weekday that fish is traditionally consumed in Germany ${ }^{(26)}$. Also, we could not revert to a biomarker of fish intake for validation 
Table 2. Prospective associations between fish consumption ${ }^{\star}$ and the risks of myocardial infarction (MI) and stroke (Hazard ratios (HR) and 95\% confidence intervals $\dagger$ )

\begin{tabular}{|c|c|c|c|c|c|c|c|c|c|c|}
\hline & \multicolumn{9}{|c|}{ Quintiles of fish consumption } & \multirow[b]{3}{*}{$P_{\text {trend }}$} \\
\hline & \multirow[b]{2}{*}{ Q1 } & \multicolumn{2}{|r|}{ Q2 } & \multicolumn{2}{|r|}{ Q3 } & \multicolumn{2}{|r|}{ Q4 } & \multicolumn{2}{|r|}{ Q5 } & \\
\hline & & $\mathrm{HR}$ & $95 \% \mathrm{Cl}$ & $\mathrm{HR}$ & $95 \% \mathrm{Cl}$ & $\mathrm{HR}$ & $95 \% \mathrm{Cl}$ & $\mathrm{HR}$ & $95 \% \mathrm{Cl}$ & \\
\hline \multicolumn{11}{|l|}{ Total MI } \\
\hline Cases $(n)$ & 138 & & 111 & & 113 & & 114 & & 129 & \\
\hline Incidence§ & 176 & & 140 & & 142 & & 144 & & 164 & \\
\hline Model 1\| & Reference & 0.93 & $0.72,1.19$ & 0.89 & $0 \cdot 70,1 \cdot 15$ & 0.86 & $0 \cdot 67,1 \cdot 10$ & 0.86 & $0 \cdot 68,1 \cdot 10$ & 0.21 \\
\hline Model 2ף & Reference & 0.91 & $0.70,1.17$ & 0.90 & $0.70,1.17$ & 0.85 & $0.66,1.09$ & 0.84 & $0.66,1.08$ & 0.21 \\
\hline \multicolumn{11}{|l|}{ Non-fatal Ml } \\
\hline Cases $(n)$ & 114 & & 94 & & 91 & & 90 & & 99 & \\
\hline Incidence§ & 145 & & 119 & & 115 & & 114 & & 126 & \\
\hline Model 1\| & Reference & 0.95 & $0.72,1.25$ & 0.87 & $0 \cdot 66,1 \cdot 15$ & 0.82 & $0.62,1.08$ & $0 \cdot 81$ & $0.62,1.07$ & 0.09 \\
\hline Model 2q & Reference & 0.91 & $0.69,1.21$ & 0.87 & $0.66,1.16$ & 0.80 & $0.60,1.06$ & 0.78 & $0.59,1.03$ & 0.07 \\
\hline \multicolumn{11}{|l|}{ Fatal MI } \\
\hline Cases $(n)$ & 24 & & 17 & & 22 & & 24 & & 30 & \\
\hline Incidence§ & 30 & & 21 & & 28 & & 30 & & 38 & \\
\hline Model 1\| & Reference & 0.82 & $0.44,1.54$ & 1.01 & $0.57,1.82$ & 1.05 & $0.59,1.85$ & $1 \cdot 11$ & $0.64,1.91$ & 0.51 \\
\hline Model 2q & Reference & 0.87 & $0.46,1.64$ & $1 \cdot 11$ & $0.62,2.02$ & $1 \cdot 13$ & $0.63,2.03$ & $1 \cdot 18$ & $0.68,2.06$ & 0.37 \\
\hline \multicolumn{11}{|l|}{ Total stroke } \\
\hline Cases $(n)$ & 103 & & 89 & & 99 & & 117 & & 117 & \\
\hline Incidence§ & 131 & & 112 & & 125 & & 148 & & 148 & \\
\hline Model 1\| & Reference & 0.88 & $0.66,1.17$ & 0.93 & $0.70,1.22$ & 1.06 & $0.81,1.38$ & 0.99 & $0.75,1.29$ & 0.66 \\
\hline Model 2ף & Reference & 0.87 & $0.65,1.16$ & 0.93 & $0.70,1.23$ & 1.05 & $0.80,1.38$ & 0.96 & $0.73,1.26$ & 0.67 \\
\hline \multicolumn{11}{|c|}{ Ischaemic stroke } \\
\hline Cases $(n)$ & 80 & & 72 & & 78 & & 92 & & 85 & \\
\hline Incidence§ & 102 & & 91 & & 98 & & 116 & & 108 & \\
\hline Model 1\| & Reference & 0.91 & $0.66,1.26$ & 0.93 & $0.68,1.27$ & 1.06 & $0.78,1.43$ & 0.91 & $0.67,1.24$ & 0.80 \\
\hline Model 2ף & Reference & 0.88 & $0.64,1.22$ & 0.92 & $0.67,1.26$ & 1.03 & $0.75,1.40$ & 0.87 & $0.64,1.19$ & 0.66 \\
\hline \multicolumn{11}{|c|}{ Haemorrhagic stroke } \\
\hline Cases $(n)$ & 16 & & 15 & & 19 & & 20 & & 25 & \\
\hline Incidence§ & 20 & & 19 & & 24 & & 25 & & 32 & \\
\hline Model 1\| & Reference & 0.99 & $0.49,2.0$ & 1.20 & $0.61,2.34$ & 1.24 & $0.64,2 \cdot 40$ & 1.44 & $0.77,2 \cdot 71$ & 0.18 \\
\hline Model 2ף & Reference & 1.0 & $0.49,2.04$ & 1.23 & $0.62,2.43$ & 1.27 & $0.65,2.51$ & 1.46 & $0.77,2.78$ & 0.16 \\
\hline
\end{tabular}

${ }^{*}$ Fish consumption was adjusted for energy by the residual method.

† Calculated by Cox proportional hazards regression analyses.

$\ddagger$ Linear trends assessed by assigning fish consumption quintile medians.

$\S$ Incidence rate per 100000 person-years.

|| Model 1: stratified by age at baseline and study centres, adjusted for sex.

I Model 2: as model 1, additionally adjusted for energy intake, alcohol intake, BMI, waist circumference, physical activity, educational attainment, smoking and prevalent diabetes mellitus.

purposes. As a nested case-control study from northern Sweden has recently shown that erythrocyte concentrations of mercury, a biomarker that is relatively specific for fish intake, were inversely associated with the risk of MI, while there was no association with FFQ-derived fish consumption levels ${ }^{(34)}$, we cannot exclude that the lack of associations in the present study was due to the limited relative validity of the FFQ. Finally, it cannot be ruled out that fish consumption in the present study changed over time. Yet, there was no indication for an interaction of fish consumption and time on study. Excluding participants with prevalent cancer, prevalent diabetes or a diagnosis of MI or stroke within the first 2 years of follow-up did not affect the results of the analyses.

As hypothesised, we only observed a weak trend for an inverse association between fish consumption and the risk of non-fatal MI. This finding is in line with previous studies that have not shown clear associations between fish consumption and the risk of non-fatal $\mathrm{CHD}^{(6,11)}$. In contrast to our hypothesis and the results of a recent meta-analysis that has confirmed a strong inverse relationship between fish consumption and CHD mortality ${ }^{(8)}$, we detected no inverse association between fish consumption and the risk of fatal MI. It is noteworthy, however, that this meta-analysis revealed substantial heterogeneity in the association between moderate fish intake and fatal CHD risk among European studies $\left(I^{2}=71 \%\right)$ and that a pooled analysis of European studies showed no significant association ${ }^{(8)}$. Further, the inverse associations between fish consumption and fatal $\mathrm{CHD}$ risk tended to be weaker in studies published after 2004 when compared with older studies ${ }^{(8)}$. This may be explained by the increased contamination of fish with pollutants and the change in nutrient composition of farmed fish, as currently discussed with regard to the lack of association between fish intake and diabetes risk ${ }^{(35)}$. Admittedly, this explanation remains speculative, as we cannot provide evidence on changes in pollutant and nutrient profiles of fish consumed in Germany from the present study or other studies. It has been proposed that the inverse association between CHD mortality and fish consumption could be driven by a decreased risk of sudden cardiac death, rather 
Table 3. Prospective associations between fish consumption* and the risk of myocardial infarction (MI) and stroke by educational attainment strata† (Hazard ratios (HR) and $95 \%$ confidence intervals $\ddagger$ )

\begin{tabular}{|c|c|c|c|c|c|c|c|c|c|c|}
\hline & \multicolumn{9}{|c|}{ Quintiles of fish consumption } & \multirow[b]{3}{*}{$P_{\text {trend }} \S$} \\
\hline & \multirow[b]{2}{*}{ Q1 } & \multicolumn{2}{|r|}{ Q2 } & \multicolumn{2}{|r|}{ Q3 } & \multicolumn{2}{|r|}{ Q4 } & \multicolumn{2}{|r|}{ Q5 } & \\
\hline & & HR & $95 \% \mathrm{Cl}$ & HR & $95 \% \mathrm{Cl}$ & $\mathrm{HR}$ & $95 \% \mathrm{Cl}$ & $\mathrm{HR}$ & $95 \% \mathrm{Cl}$ & \\
\hline \multicolumn{11}{|l|}{ MI } \\
\hline \multicolumn{11}{|c|}{ Low education ( $n$ 11275) } \\
\hline Cases $(n)$ & 55 & & 39 & & 37 & & 38 & & 38 & \\
\hline Incidence\| & 288 & & 218 & & 208 & & 210 & & 212 & \\
\hline \multicolumn{10}{|c|}{ Medium education ( $n$ 20 564) } & 0.09 \\
\hline Cases $(n)$ & 48 & & 35 & & 38 & & 42 & & 51 & \\
\hline \multirow[t]{2}{*}{ Incidence $\|$} & 146 & & 101 & & 112 & & 123 & & 156 & \\
\hline & \multicolumn{9}{|c|}{ High education ( $n$ 16 476) } & 0.78 \\
\hline Cases $(n)$ & 35 & & 37 & & 38 & & 34 & & 40 & \\
\hline \multirow[t]{2}{*}{ Incidence $\|$} & 132 & & 139 & & 137 & & 125 & & 142 & \\
\hline & Reference & $1 \cdot 14$ & $0.72,1.83$ & $1 \cdot 11$ & $0.69,1.77$ & 0.91 & $0.57,1.48$ & 0.91 & $0.57,1.44$ & 0.40 \\
\hline \multirow{2}{*}{\multicolumn{11}{|c|}{$\begin{array}{l}\text { Stroke } \\
\quad \text { Low education ( } n 11275)\end{array}$}} \\
\hline \multicolumn{8}{|c|}{ Low education ( $n$ 11275) } & & & \\
\hline Cases $(n)$ & 39 & & 33 & & 33 & & 35 & & 35 & \\
\hline \multirow{2}{*}{ Incidence\|l } & 204 & & 184 & & 186 & & 194 & & 195 & \\
\hline & \multicolumn{10}{|c|}{ Medium education ( $n$ 20 564) } \\
\hline Cases $(n)$ & 42 & & 37 & & 39 & & 49 & & 39 & \\
\hline \multirow[t]{2}{*}{ Incidence\|l| } & 128 & & 106 & & 116 & & 144 & & 119 & \\
\hline & Reference & 0.87 & $0.55,1.36$ & 0.86 & $0.55,1.34$ & 1.04 & $0.68,1.59$ & 0.80 & $0.51,1 \cdot 25$ & 0.53 \\
\hline & 22 & & 19 & & 27 & & 33 & & 43 & \\
\hline \multirow[t]{2}{*}{ Incidence $\|$} & 83 & & 71 & & 97 & & 122 & & 153 & \\
\hline & Reference & 0.80 & $0.43,1.49$ & 1.07 & $0.60,1.90$ & 1.25 & $0.72,2.17$ & 1.42 & $0.84,2.39$ & 0.04 \\
\hline
\end{tabular}

${ }^{*}$ Fish consumption was adjusted for energy by the residual method.

† Model stratified by age at baseline and study centres, adjusted for sex, energy intake, alcohol intake, BMI, waist circumference, physical activity, smoking and prevalent diabetes mellitus.

$\ddagger$ Calculated by Cox proportional hazards regression analyses.

$\S$ Linear trends assessed by assigning fish consumption quintile medians.

|| Incidence rate per 100000 person-years.

than a decreased risk of other CHD events ${ }^{(36)}$. Therefore, another possible explanation for the lack of association between fish consumption and fatal MI in the present study could be our definition of a fatal MI that included a rather long period of $28 \mathrm{~d}$ between event and death. Actually, there was no association between fish intake and the risk of fatal MI in one cohort study that also applied the $28 \mathrm{~d}$ rule ${ }^{(37)}$, although one further study did show a significant inverse association between fish consumption and fatal MI within $28 \mathrm{~d}$ after disease incidence ${ }^{(38)}$.

Unlike the present study, a recent meta-analysis showed a significant stroke risk decrease by $14 \%$ at a moderate habitual fish intake of one serving/week compared with an intake of less than one serving $/$ month $^{(9)}$. Another recent meta-analysis that also showed an overall inverse association between fish consumption and stroke risk revealed that this association was weaker and no longer significant when pooling studies characterised by lower average fish consumption levels, i.e. levels below $200 \mathrm{~g} /$ week $^{(10)}$. Interestingly, in line with the present results, no significant association between fish consumption and stroke risk was shown in the former meta-analysis pooling study results from Europe ${ }^{(9)}$, whereas the latter one did provide an overall significant inverse association from the same original studies ${ }^{(10)}$. While the former meta-analysis further indicated that the protective potential of fish consumption might be restricted to ischaemic strokes $^{(9)}$, we found no association between fish consumption and the risk of ischaemic stroke.

Tests for interaction in the present study suggested differential associations between fish consumption and the risks of MI and stroke depending on the participants' education level. Analyses stratified by education level showed a modest trend for a positive association concerning stroke risk in subjects with a high education level and a weak non-significant trend for an inverse association concerning MI risk in subjects with a low education level. Yet, there is no evidence on socio-economic differences in relation to fish consumption and CVD risk from the literature. Also, we had no information on preferred fish types and preparation methods according to education level, neither from the present study nor from other studies in German populations. Thus, the present finding of interactions between fish consumption and education level may be due to chance.

In summary, fish consumption was not associated with lower risks of MI and stroke in the EPIC-Germany study. We could not reproduce previous study results suggesting that fish consumption protects from fatal CHD and ischaemic stroke. The present results might indicate that associations 
between fish consumption and the risks of MI and stroke are weaker in populations with low overall fish intakes, despite the notion from recent meta-analyses that the risks of fatal CHD and stroke may significantly decrease even at intakes of one serving of fish per week.

\section{Acknowledgements}

The present study is part of the joint project 'Vitamin D and Cardiovascular Health' funded by the German Federal Ministry of Education and Research (grant no. 0315668). C. W., B. T., B. B., H. B. and R. K. were responsible for the data acquisition. T. K., B. T., C. W., R. K. and B. B. were responsible for the data evaluation concept. T. K. conducted the statistical analyses and drafted the manuscript. All authors interpreted the data, and critically revised and approved the manuscript. None of the authors declares a conflict of interest.

\section{References}

1. Beaglehole R \& Bonita R (2008) Global public health: a scorecard. Lancet 372, 1988-1996.

2. Franco M, Cooper RS, Bilal U, et al. (2011) Challenges and opportunities for cardiovascular disease prevention. $\mathrm{Am} \mathrm{J}$ Med 124, 95-102.

3. World Health Organization (2003) Diet, Nutrition and the Prevention of Chronic Diseases. Joint WHO/FAO Expert Consultation. WHO Technical Report Series no. 916. Geneva: WHO.

4. Lloyd-Jones DM, Hong Y, Labarthe D, et al. (2010) Defining and setting national goals for cardiovascular health promotion and disease reduction: the American Heart Association's strategic Impact Goal through 2020 and beyond. Circulation 121, 586-613.

5. Kris-Etherton PM, Grieger JA \& Etherton TD (2009) Dietary reference intakes for DHA and EPA. Prostaglandins Leukot Essent Fatty Acids 81, 99-104.

6. He K (2009) Fish, long-chain omega-3 polyunsaturated fatty acids and prevention of cardiovascular disease - eat fish or take fish oil supplement? Prog Cardiovasc Dis 52, 95-114.

7. Mente A, de Koning L, Shannon HS, et al. (2009) A systematic review of the evidence supporting a causal link between dietary factors and coronary heart disease. Arch Intern Med 169, 659-669.

8. Zheng J, Huang T, Yu Y, et al. (2012) Fish consumption and CHD mortality: an updated meta-analysis of seventeen cohort studies. Public Health Nutr 15, 725-737.

9. Xun P, Qin B, Song Y, et al. (2012) Fish consumption and risk of stroke and its subtypes: accumulative evidence from a meta-analysis of prospective cohort studies. Eur J Clin Nutr 66, 1199-1207.

10. Chowdhury R, Stevens S, Gorman D, et al. (2012) Association between fish consumption, long chain omega 3 fatty acids, and risk of cerebrovascular disease: systematic review and meta-analysis. BMJ 345, e6698-e6698.

11. Mozaffarian D \& Wu JH (2011) Omega-3 fatty acids and cardiovascular disease: effects on risk factors, molecular pathways, and clinical events. I Am Coll Cardiol 58, 2047-2067.

12. Brouwer IA, Geelen A \& Katan MB (2006) n-3 Fatty acids, cardiac arrhythmia and fatal coronary heart disease. Prog Lipid Res 45, 357-367.
13. Cottin SC, Sanders TA \& Hall WL (2011) The differential effects of EPA and DHA on cardiovascular risk factors. Proc Nutr Soc 70, 215-231.

14. de Goede J, Geleijnse JM, Boer JM, et al. (2010) Marine (n-3) fatty acids, fish consumption, and the 10-year risk of fatal and nonfatal coronary heart disease in a large population of Dutch adults with low fish intake. J Nutr 140, 1023-1028.

15. Mozaffarian D (2008) Fish and $n-3$ fatty acids for the prevention of fatal coronary heart disease and sudden cardiac death. Am J Clin Nutr 87, 1991S-1996S.

16. De Caterina R (2011) $n$-3 Fatty acids in cardiovascular disease. $N$ Engl J Med 364, 2439-2450.

17. Grant WB, Cross HS, Garland CF, et al. (2009) Estimated benefit of increased vitamin $\mathrm{D}$ status in reducing the economic burden of disease in western Europe. Prog Biophys Mol Biol 99, 104-113.

18. Pilz S, Tomaschitz A, Marz W, et al. (2011) Vitamin D, cardiovascular disease and mortality. Clin Endocrinol (Oxf) $\mathbf{7 5}$, 575-584.

19. Turunen AW, Mannisto S, Suominen AL, et al. (2011) Fish consumption in relation to other foods in the diet. $\mathrm{BrJ}$ Nutr 106, 1570-1580.

20. Darmon N \& Drewnowski A (2008) Does social class predict diet quality? Am J Clin Nutr 87, 1107-1117.

21. Elmadfa I \& Kornsteiner M (2009) Fats and fatty acid requirements for adults. Ann Nutr Metab 55, 56-75.

22. Failler P (2007) Future prospects for fish and fishery products. 4. Fish consumption in the European Union in 2015 and 2030. Part 1. European overview. In FAO Fisheries Circular, pp. 20-21. Rome: FAO.

23. Jakobsen MU, Dethlefsen C, Due KM, et al. (2013) Fish consumption and subsequent change in body weight in European women and men. Br J Nutr 109, 353-362.

24. Boeing H, Wahrendorf J \& Becker N (1999) EPIC-Germany a source for studies into diet and risk of chronic diseases. European Investigation into Cancer and Nutrition. Ann Nutr Metab 43, 195-204.

25. Bergmann MM, Bussas U \& Boeing H (1999) Follow-up procedures in EPIC-Germany - data quality aspects. European Prospective Investigation into Cancer and Nutrition. Ann Nutr Metab 43, 225-234.

26. Bohlscheid-Thomas S, Hoting I, Boeing H, et al. (1997) Reproducibility and relative validity of food group intake in a food frequency questionnaire developed for the German part of the EPIC project. European Prospective Investigation into Cancer and Nutrition. Int J Epidemiol 26, Suppl. 1, S59-S70.

27. Brandstetter BR, Korfmann A, Kroke A, et al. (1999) Dietary habits in the German EPIC cohorts: food group intake estimated with the food frequency questionnaire. European Investigation into Cancer and Nutrition. Ann Nutr Metab 43, 246-257.

28. Nagel G, Zoller D, Ruf T, et al. (2007) Long-term reproducibility of a food-frequency questionnaire and dietary changes in the European Prospective Investigation into Cancer and Nutrition (EPIC)-Heidelberg cohort. BrJ Nutr 98, 194-200.

29. Tunstall-Pedoe H, Kuulasmaa K, Mahonen M, et al. (1999) Contribution of trends in survival and coronary-event rates to changes in coronary heart disease mortality: 10-year results from 37 WHO MONICA project populations. Monitoring trends and determinants in cardiovascular disease. Lancet 353, 1547-1557.

30. Willett WC, Howe GR \& Kushi LH (1997) Adjustment for total energy intake in epidemiologic studies. Am J Clin Nutr $\mathbf{6 5}$, 1220S-1228S, discussion 1229S-1231S. 
31. Wareham NJ, Jakes RW, Rennie KL, et al. (2003) Validity and repeatability of a simple index derived from the short physical activity questionnaire used in the European Prospective Investigation into Cancer and Nutrition (EPIC) study. Public Health Nutr 6, 407-413.

32. Heinzl H \& Kaider A (1997) Gaining more flexibility in Cox proportional hazards regression models with cubic spline functions. Comput Methods Programs Biomed 54, 201-208.

33. Durrleman S \& Simon R (1989) Flexible regression models with cubic splines. Stat Med 8, 551-561.

34. Wennberg M, Bergdahl IA, Hallmans G, et al. (2011) Fish consumption and myocardial infarction: a second prospective biomarker study from northern Sweden. Am J Clin Nutr 93, 27-36.
35. Ruzzin J \& Jacobs DR (2012) The secret story of fish: decreasing nutritional value due to pollution? Br J Nutr $\mathbf{1 0 8}$, 397-399.

36. Siscovick DS, Lemaitre RN \& Mozaffarian D (2003) The fish story. Circulation 107, 2632-2634.

37. Iso H, Kobayashi M, Ishihara J, et al. (2006) Intake of fish and $n-3$ fatty acids and risk of coronary heart disease among Japanese: the Japan Public Health Center-Based (JPHC) Study Cohort I. Circulation 113, 195-202.

38. Yuan JM, Ross RK, Gao YT, et al. (2001) Fish and shellfish consumption in relation to death from myocardial infarction among men in Shanghai, China. Am J Epidemiol 154, 809-816. 